\title{
A model to calculate consistent atmospheric emission projections and its application to Spain
}

\author{
Julio Lumbreras, Rafael Borge, Juan Manuel de Andrés, Encarnación Rodríguez \\ Department of Chemical and Entironmental Engineering, Technical University of Madrid (UPM), José Gutiérrez Abascal 2, \\ 28006 Madrid, Spain
}

\begin{abstract}
Global warming and air quality are headline environmental issues of our time and policy must preempt negative international effects with forward-looking strategies. As part of the revision of the European National Emission Ceilings Directive, atmospheric emission projections for European Union countries are being calculated. These projections are useful to drive European air quality analyses and to support wide-scale decision-making. However, when evaluating specific policies and measures at sectoral level, a more detailed approach is needed. This paper presents an original methodology to evaluate emission projections. Emission projections are calculated for each emitting activity that has emissions under three scenarios: without measures (business as usual), with measures (baseline) and with additional measures (target). The methodology developed allows the estimation of highly disaggregated multi-pollutant, consistent emissions for a whole country or region. In order to assure consistency with past emissions included in atmospheric emission inventories and coherence among the individual activities, the consistent emission projection (CEP) model incorporates harmonization and integration criteria as well as quality assurance/quality check (QA/QC) procedures. This study includes a sensitivity analysis as a first approach to uncertainty evaluation. The aim of the model presented in this contribution is to support decision-making process through the assessment of future emission scenarios taking into account the effect of different detailed technical and non-technical measures and it may also constitute the basis for air quality modelling. The system is designed to produce the information and formats related to international reporting requirements and it allows performing a comparison of national results with lower resolution models such as RAINS/ GAINS. The methodology has been successfully applied and tested to evaluate Spanish emission projections up to 2020 for 26 pollutants but the methodology could be adopted for any particular region for different purposes, especially for European countries.
\end{abstract}

Keywords: Air pollution; Model; Emission projections; Projections uncertainty; QA/QC

\section{Introduction}

It is generally recognized that effective air quality policies and measures (P\&M) can only be based on quantitative information on both current and future emissions of pollutants (Samaras et al., 1999). In 
order to evaluate these emission projections, it is important to compile technology-based emission inventories consisting of detailed activity data and representative emission factors. Considerable efforts have been made in the framework of the UNECE Convention for Long Range Transport of Air Pollution, both in the USA (USA, 2002) and the European Union (EC, 2004) to develop and refine national or regional emission inventories. The methodology used to estimate emissions in the $\mathrm{EU}$ is coordinated by the European Environment Agency through the COoRdination of INformation on AIR (CORINAIR) emission inventory, the associated methodology (EEA, 2006) and Common Reporting Format (CRF) of UNFCCC.

However, a limited amount of research has been done in developing models to forecast atmospheric emissions (Samaras et al., 1999; US EPA, 1999). Some projections of regional or global emissions have been recently done but they often do not rely on a formal approach (a model) but rather extrapolate from current trends. Moreover, if modelled they are on a larger scale or very small scale, like particular sector only. For instance, Derwent et al. (2006) projected ozone precursors for anthropogenic sectors in Ireland, Garg et al. (2004) developed future $\mathrm{CH}_{4}$ and $\mathrm{N}_{2} \mathrm{O}$ emissions from all sectors for India, Klimont et al. (2002) estimated anthropogenic NMVOC emissions in China, McCulloch and Lindley (2007) have done a global calculation of HFC-23 from HCFC-22 production until 2015, Pirrone et al. (2001) projected mercury emissions for the Mediterranean basin from major industrial sources, and Streets and Waldhow (2000) estimated $\mathrm{SO}_{2}, \mathrm{NO}_{x}$ and $\mathrm{CO}$ for all China for year 2020. In this paper, a highly detailed consistent emission projection (CEP) model is developed to address national emission projections under a multi-pollutant and multi-sector approach. In contrast with previous studies, this methodology encompasses all major pollutants (26) and activity sectors (282) covering both anthropogenic and natural emission sources at national level. The model has been applied and tested for Spain in the period 2001-2020.

Although national emission projections have become a requirement for EU Member States and are being included in the integrated assessment modelling process conducted under the Clean Air For Europe (CAFE) programme and in the Revision of the National Emission Ceilings (NEC)
Directive (2001/81/EC), there is still a lack of clearly stated procedures and methodologies for making national emission projections suitable for meeting international information requirements while keeping full consistency with national atmospheric emission inventories. The CEP model provides a sound methodology for emission projections in this scope. One of its main objectives is to provide information on future emissions of pollutants included in the Kyoto Protocol (UNFCCC, 1997) and the Geneva Convention (GC, 1979). Other specific objectives of the model are as follows:

- Determine future emission scenarios based on alternative policies and/or technologies.

- Estimate the effectiveness of the abatement measures included under each scenario.

- Assess the compliance with international agreements such as the Kyoto Protocol and the NEC Directive.

The application of this methodology produced annual emissions of greenhouse effect gases (GHG), acidifying gases, heavy metals, particulate matter and ozone precursors for Spain up to the year 2020 . These projections have been developed under several scenarios, compatible with the CAFE methodology. The flowchart in Fig. 1 provides a basic picture of the model including data types, sources, main outputs and their purpose.

The fundamentals and rationale of the projection methodology is summarized in Section 2 while the main results from the application of the model to Spain are presented in Section 3. Section 4 summarizes discussions of the methodology and implications and Section 5 presents the conclusions and outlook for future work.

\section{Methodology}

\subsection{Pollutants}

The methodology considers the 11 pollutants included in the Geneva Convention and the 15 gases under the Kyoto Protocol.

\subsection{Activities considered}

The activities considered are those included in the Selected Nomenclature for Air Pollution (SNAP 97) within the CORINAIR methodology. The SNAP system has a hierarchical structure of three levels 




Fig. 1. CEP framework. The CEP model includes all calculation methods (yellow charts), procedures (green charts), software tool (violet chart) and results (red charts) within the orange coloured box on the top. Exogenous inputs are placed on the bottom-left part of the figure distinguishing between the NAEI (green colour) and envisaged policies and measures (blue). Reporting endpoints and purpose of model outputs are illustrated on the lower-right side.

called Group, Sub-group and Activity (EEA, 2006) related to both, anthropogenic and natural emission sources.

Two basic classes of emission sources have been considered, point and area sources. Area sources comprise various emission-producing units which, as a result of their small individual significance or due to the way their basic data are represented (breakdown by territorial units), must be dealt with on an aggregate basis for a particular geographical area. Point sources are those which, owing to their high contribution to total emissions and their release conditions, must be dealt with individually. The criteria used to identify large point sources (LPS) are those proposed in the CORINAIR methodology (EEA, 2006). This issue provides useful information for modelling purposes and enables the integration of plant-specific plans and regulations.

\subsection{Emission projection calculations}

When projecting atmospheric emissions to future years using the CEP methodology, one of the basic principles is to be fully consistent with the official past emission series, usually referred to as National Atmospheric Emission Inventory (NAEI). Consequently, data sources and computation methods involved in the compilation of NAEI are extended to projected series. For instance, for the Spanish case, $\mathrm{NH}_{3}$ emission projections from livestock were calculated using the same assumptions on number of housing days, amount of nitrogen excreted in housing and grazing (in $\mathrm{kg}$ of $\mathrm{N} / \mathrm{animalyear}$ ) and the same volatilization percentage for housing, storage, application, and grazing. However, in certain cases an improved approach could be implemented, e.g., when more detailed information on a specific sector not included in NAEI has 
become available. A common example of this situation can be found in the industrial sector, where Best Available Techniques (BAT) shall be applied. For all the industrial activities included in the inventory (SNAP groups 3 and 4), penetrations of BAT were considered according to national guidelines and interviews with sectoral experts.

As a result, the top-down approach was used for $90 \%$ of the different activities (252 out of 282) included in the CEP for the Spanish case. Nevertheless, the remaining $10 \%$ has a very significant impact on national emission levels and were calculated using a bottom-up approach (MMA, 2006). In addition, the bottom-up approach was used to quantify future emissions from smaller point sources for which specific legislation applies, such as some activities included in the solvent use sector (SNAP group 6).

Regardless of the aggregation level, emission projections were calculated using two different methodological approaches depending on the information available: (a) the technology-based approach and (b) the surrogate growth approach. The rationale and algorithms behind each option are explained in Sections 2.3.1 and 2.3.2. The computation methods applied are consistent with the general approach of US EPA (1999) and van Harmelen and Pulles (2000). All projections were estimated on an annual, national basis (except for LPS dealt with individually) at the highest possible detail, which means the most detailed source-sector level included in the SNAP reporting nomenclature used within the EU and UN air pollution regulation (documented in EEA, 2006).

\subsubsection{Technology-based projections}

This approach was used when detailed information about future activity rates $\left(A_{i}\right)$ and technological changes were either available or predictable. This includes implementation of end-of-pipe emission control measures, technological improvements in existing production processes and penetration of emerging production technologies as well as predicted trends of activity growth. Projection calculations were carried out for each activity as follows:

$E_{i, j}=A_{i} \mathrm{EF}_{a, j} \prod_{k=1}^{n} \mathrm{CFt}_{j, k}$,

where $E_{i, j}$ is the emission of pollutant $j$ in year $i, A_{i}$ is the activity rate for the year $i, \mathrm{EF}_{a, j}$ is the emission factor of pollutant $j$ for the base year $a$, and $\mathrm{CFt}_{j, k}$ is the control factor for pollutant $j$ using measure $k$ shown in Eq. (2):

$\mathrm{CFt}_{j, k}=1-R_{k} P_{k}$,

where $R_{k}$ is the emission reduction rate associated with the application of measure $k$ (e.g., implementation of electrostatic precipitators for the reduction of particulate matter emissions from coal burning power plants) and $P_{k}$ is the penetration of measure $k$ (e.g., percentage of coal burnt in power plants where electrostatic precipitators are implemented). $R_{k}$ and $P_{k}$ range between 0 and 1 .

\subsubsection{Surrogate growth-based projections}

The surrogate growth approach was used when there was insufficient information to apply the method described in Eq. (1). In this case, a surrogate variable, such as gross domestic product (GDP), population, or fuel consumption was used as follows:

$E_{i, j}=G_{a-i} E_{a, j} \prod_{k=1}^{n} \mathrm{CFg}_{j, k}$,

where $E_{a_{j} j}$ is the emission of pollutant $j$ for the base year $a, G_{a-i}$ is the emission growth factor between the base year and year $i$, and $\mathrm{CFg}_{j, k}$ is the control factor for pollutant $j$ associated with the application of measure $k$. For instance, emissions from use of $\mathrm{N}_{2} \mathrm{O}$ in anaesthesia were projected using growth population as $G_{a-i}$ or forestry machinery emissions were calculated through fuel consumption and expected forest growth as surrogate variables.

Although total emissions from certain activity sectors were calculated using specific methodologies and software tools (e.g., COPERT III for road transport emissions, EEA, 2000), composite emission factors were also derived for these activities so that parameters involved in Eq. (1) were obtained. This provides a consistent framework for the analysis of emission projections from different activity sectors, facilitating thus the comparison of alternative emission scenarios within the CAFE programme.

It should be noted that emission drivers are exogenous variables to the model, i.e., the methodology does not include any econometric model to forecast basic socio-economic variables driving the activity rates or growth factors. This approach enables the model to integrate, where available, future national activity rate estimates for all sectors in a consistent manner. 


\subsection{Scenarios}

For a given activity, it is possible to define as many emission scenarios as the number of different combinations of any possible values of the parameters included in Eqs. (1) or (3). These values (e.g., $\mathrm{EF}, A, \mathrm{CFt}$, etc.) depend on socio-economic, legislative, and technological aspects and should be consistent through each scenario. The set of hypotheses made for all the parameters involved in the emission calculations determines a scenario.

In order to standardize the definition of activity hypothesis and assure global consistency, three scenarios relevant to air quality management have been adopted as discussed below.

\subsubsection{Business as usual (BAU) or without measures}

BAU scenario relies on statistical methods to project activity rates and/or emission factors to a future year, taking only into consideration past emission data and current trends. It excludes all P\&M implemented, adopted or planned after the base year. This scenario shows how emissions from a specific activity sector would grow in the absence of any additional technical or non-technical control measures.

Estimates are built upon the best regression adjustment from available past activity data (since 1990 in the Spanish case) (i.e., the regression that minimizes the non-explainable residues of the model). When long data series are available, a statistical model for short-term forecasting (Univariate Stochastic ARIMA Model) that incorporates both autoregressive parameters and the variance of the error term was used. In the few cases where no detailed information on past activity rates and emission factors was available, an emission trend was extrapolated from past emission values.

\subsubsection{Baseline scenario or with measures}

Baseline scenario is intended to provide national emission projections in consistence with the homonymous scenario defined in the CAFE Programme. It provides estimates for national emissions according to the implemented P\&M for reducing emissions through technology improvements and dissemination, demand-side efficiency gains, more efficient regulatory procedures, and shifts to cleaner fuels. This scenario has also been defined taking into consideration all sectoral plans and measures (enacted and in force) published by official organi- zations and the national sectoral legislation. Thereby, it outlines a likely range for future emissions of air pollutants and greenhouse gases.

The quantification of the effect of P\&M in emission reductions (estimated from the difference between baseline and BAU scenario) is critical for assessing their effectiveness and therefore, to guide the proposal of additional measures to achieve emission objectives. In addition, it constitutes the basis for extended cost-benefit analysis.

\subsubsection{Target scenario or with additional measures}

The target scenario incorporates additional measures in relation to the baseline scenario (measures that could also be adopted for the fulfillment of commitments under the NEC Directive and the Kyoto Protocol). These measures are based on technical references available for each sector, as for instance, the documents prepared by the Expert Group on Techno Economic Issues (CITEPA, 2003). When there is no future technology available and a change in the activity rate is economically and technically practical, it includes assumptions about alternative activity rate evolution patterns. That means that non-technical measures (e.g., efficiency improvement, consumption or production cutback) or policy instruments (e.g., fiscal and prescriptive measures), currently being discussed in Europe (IVL, 2006), may be adopted to reach the targets.

Sectoral and spatial disaggregation of emission thresholds is one of the most challenging issues for their implementation. Sectoral disaggregation is done by establishing an "emission bubble", i.e., the allowed amount of emissions for each activity for the year 2010 (at SNAP-3 level), which is proportional to the activity emission level in the base year 2000. This criterion (based on fixed emissions in 2000) is considered to be the most appropriate because it is not dependent on the evolution of emissions from each activity sector after the base year. This is a critical aspect of the proposed methodology, since the determination of the target scenario is very sensitive to activityspecific thresholds setting.

\subsubsection{Emission calculation}

Fig. 2 shows the general procedure for emission calculation carried out at activity level. The first step consists of selecting the appropriate calculation method from those explained in Section 2.3. The best available data allow defining the parameters involved in the calculation method (step 2) and are 


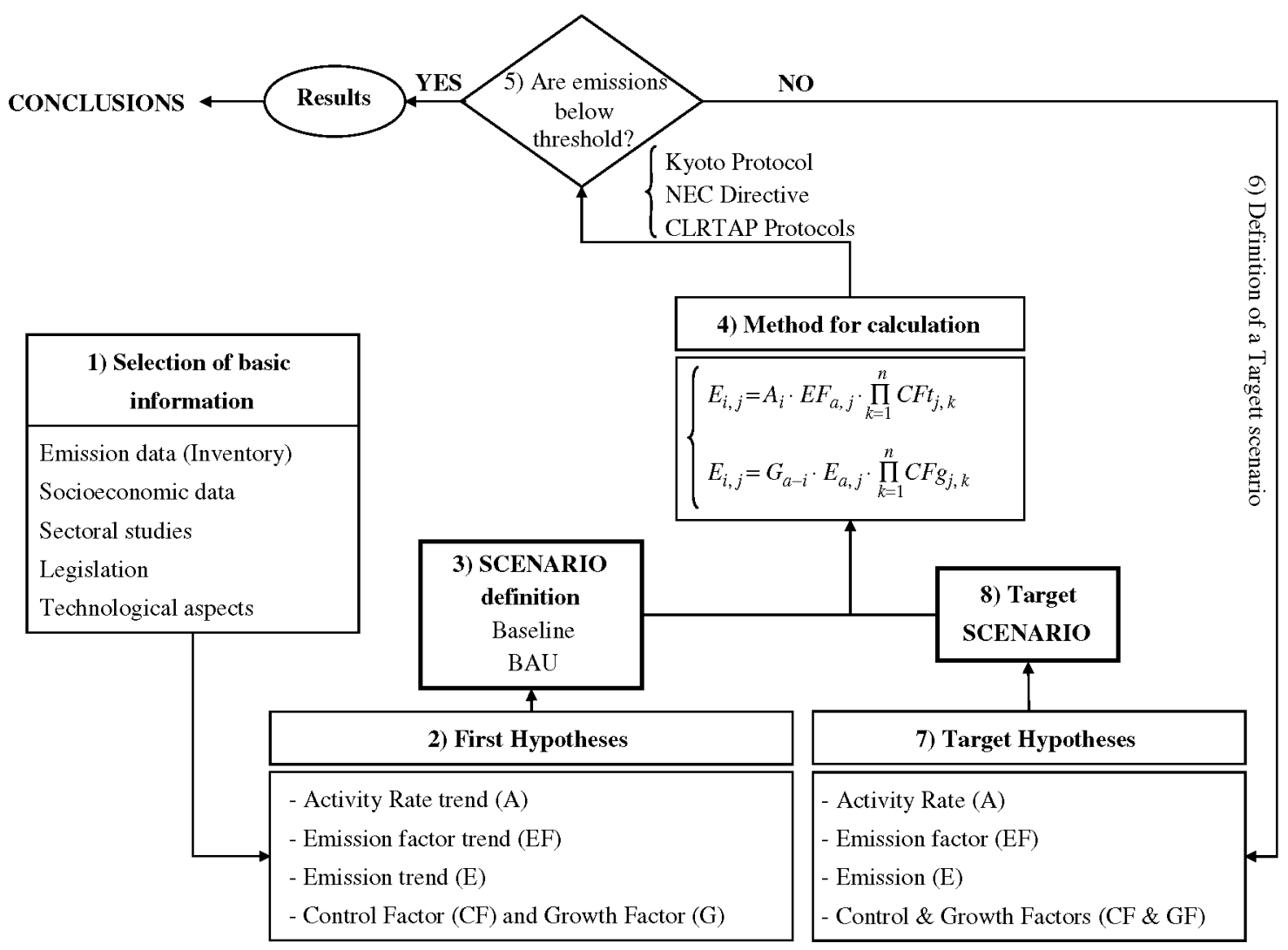

Fig. 2. General scheme for developing emission projections at activity level.

used in the characterization of BAU and baseline scenarios (step 3). Afterwards, the formulae are applied to evaluate emission projections (step 4). Results from step 4 are compared against the defined activity-level thresholds (step 5). If all pollutant projections under the baseline scenario are below the limit (for the reference year; 2010 in the Spanish case), the procedure finishes. Otherwise, a target scenario including additional measures for further reductions is introduced, and consequently, new values for main calculation parameters in Eqs. (1) or (3) (step 7) defining the target scenario (step 8) are applied. At this stage, the criterion to select the additional measures is based on their effectiveness and the interest from the decision makers (for Spain, the Ministry of Environment). However, a multi-criteria analysis including emission reductions and cost is being developed. The scheme is iterated until target emissions are below the thresholds. Supervision is needed to assure measures feasibility and avoid unrealistic assumptions.

As a consequence of reporting requirements, some spatial disaggregation was needed. In addi- tion, EEA, LRTAP Convention (EMEP, 2004) and UNFCC establish a different scope as far as pollutant datasets and emitting activities are regarded. In order to reduce computational requirements, a parallel database scheme was adopted. Further discussion on this issue can be found in Borge et al. (2005).

\subsection{Integration of scenarios}

For integration purposes, all the activities identified as having a clear connection through the activity rates involved in the emission estimation are gathered in a "Macroscenario", as it is shown in Fig. 3. This concept is discussed in Section 4.1. The same name is also applied to the joint projection of these groups of activities resulting from the addition of individual projections. For instance, the energy generation scenario that includes combustion in energy and transformation industries except district heating plants, non-industrial combustion plants, and electrical equipments (except electronic components manufacturing). Macroscenarios for industry, 


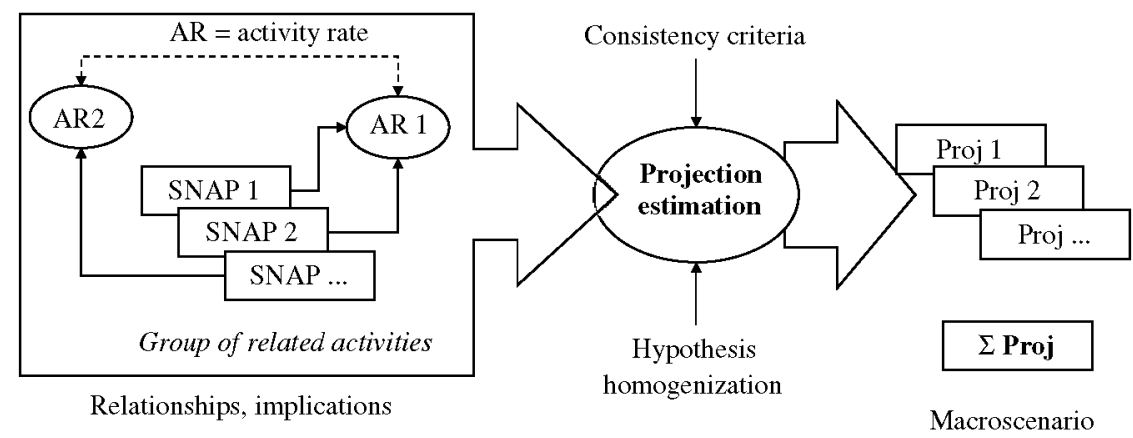

Fig. 3. The macroscenario concept and individual results merging process.

transport, waste management, livestock-agriculture and nature were also defined. Once the relationship mapping, (i.e., the connection between activities as indicated in Fig. 3), has been defined, the completion of the method is only a matter of introducing consistency criteria into the hypothesis made under each scenario for a particular activity rate. For those activities using the same or strongly related activity rates, consistent values are considered across all the SNAP activities involved.

A software tool (EmiPro) has been developed as part of this model to support the macroscenario definition and the emission projection grouping. EmiPro implements the methodology described above and facilitates reporting and comparison with other emission projection methodologies, such as RAINS. Its main functionalities are: (i) storage and recovery of past emissions, (ii) generation of projections from history data and algorithm factors, (iii) storage and recovery of projected emissions, (iv) reports generation, and (v) quality assurance/ quality check (QA/QC) support. A detailed description can be found in Borge et al. (2005).

\section{6. $Q A / Q C$ of calculations}

The QA/QC procedure applied in projections consists of automatic checking of intermediate results, such as the use of different fuels in an industrial process to assure coherence between fuel consumption, technological innovation and production rates. In addition, emission projections are calculated retrospectively for past years and compared with the values included in the SNAEI for all pollutants.

When emission projections are calculated statistically from past values, the predicted emissions are checked to ensure that unrealistic values, such as negative emissions, are replaced by adequate values derived from reliable emission factors and/or activity rates (e.g., asymptotic reduction scenario).

Furthermore, a double-checking mechanism based on global fuel consumption is implemented within CEP. This mechanism allows the comparison between the aggregated fuel consumption from all activities (calculated using both bottom-up and topdown methodologies) and the total national fuel consumption projected by the official national institute (in the Spanish case, the Ministry of Industry MICYT, 2003) and international fuel consumption projections (Capros and Mantzos, 2004; Amann et al., 2007). This fuel budget provides values for each sector and type of fuel used in the three scenarios (BAU, baseline and target). All together, 28 different types of fuel are further grouped into different national and international fuel categories. Finally, the fuel budget mechanism is implemented into the software tool EmiPro, which can be used to detect and fix possible inconsistencies in the emission projections.

EmiPro also provides warnings intended to alert the user on inappropriate or suspicious results such as large leaps in emissions for consequent years or greater emission values for $\mathrm{PM}_{2.5}$ than $\mathrm{PM}_{10}$.

\section{Model application to Spain}

This section presents the input data and the main assumptions considered to evaluate Spanish emission projections applying the CEP model.

\subsection{Business as usual scenario}

The BAU scenario is used to figure out the impact of $\mathrm{P} \& M$ included in the baseline scenario in emission reduction. Fig. 4 presents three illustrative examples of the alternatives for computation. The 
a

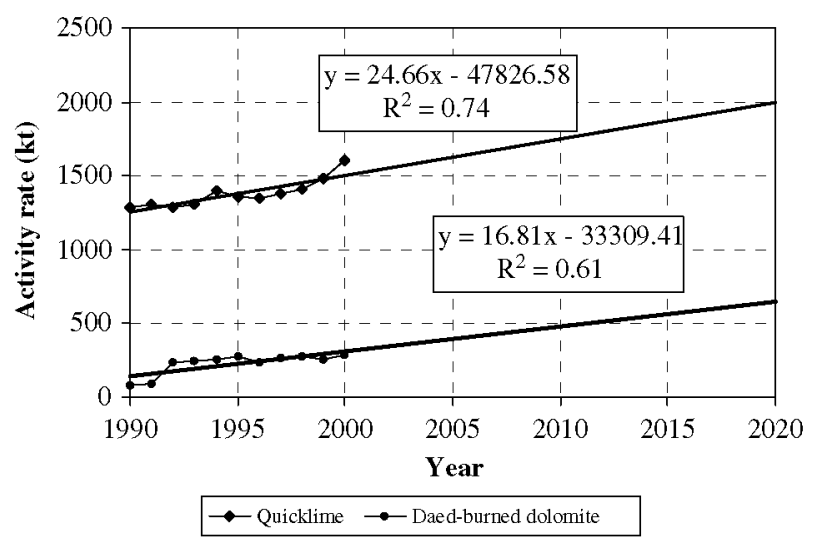

b

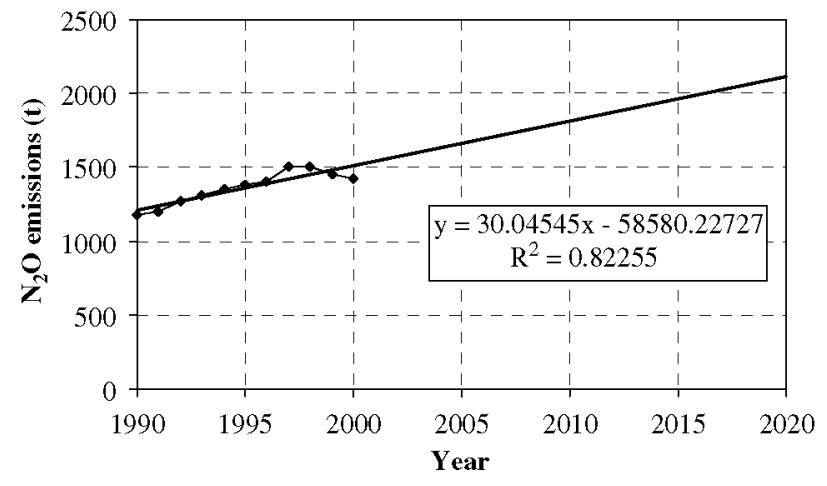

C

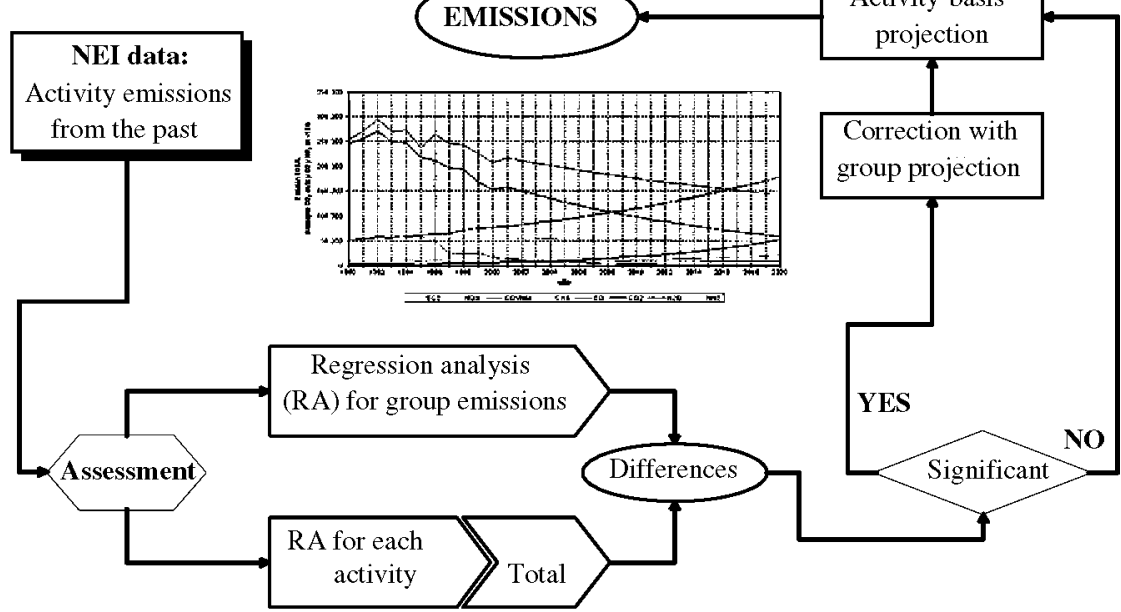

Fig. 4. Examples of BAU scenario types of calculation.

first two graphs show regression adjustments for activity rate (Fig. 4a for lime production-quicklime and daed-burned dolomite) and emission data (Fig. 4b for $\mathrm{N}_{2} \mathrm{O}$ emissions from anesthesia applications). This method is used when reasonable correlation values are obtained $\left(R^{2}>0.6\right)$. In some cases, when a group of activity are closely related, it is advisable to get an aggregated tendency, so individual projections can be merged in a meaningful way. This approach was applied for road transport activities as shown in Fig. 4c.

Fig. 5 shows national-aggregated emission projections for $\mathrm{CO}_{2}$ (Fig. 5a), $\mathrm{CO}_{2}$ equivalent (Fig. 5b), and the NEC pollutants. All these gases show a substantial increase under the BAU scenario. The highest increment for the period 2000-2020 corresponded to $\mathrm{SO}_{2}$ and $\mathrm{CO}_{2}(+88 \%$ and $+83 \%)$ due to the absence of technical measures to abate emissions and to the tendency of energy consumption observed in the past, respectively.

\subsection{Baseline scenario}

As a starting point for further analysis Table $1 \mathrm{~s}$, included as supplementary material, presents the main P\&M considered in the baseline scenario at SNAP-group level. The table includes the name of each measure, the sectors affected, targeted pollutants and a relevant reference.

Table 1 summarizes the effect of the adoption of the measures mentioned above in reference to BAU scenario. Measures devised for the agriculture sector are the most effective for total $\mathrm{NH}_{3}$ reductions (they would reduce $52 \mathrm{kt}$, which means $80 \%$ of national $\mathrm{NH}_{3}$ abatement). Concerning NMVOC, technological measures for on-road transport could save 
a

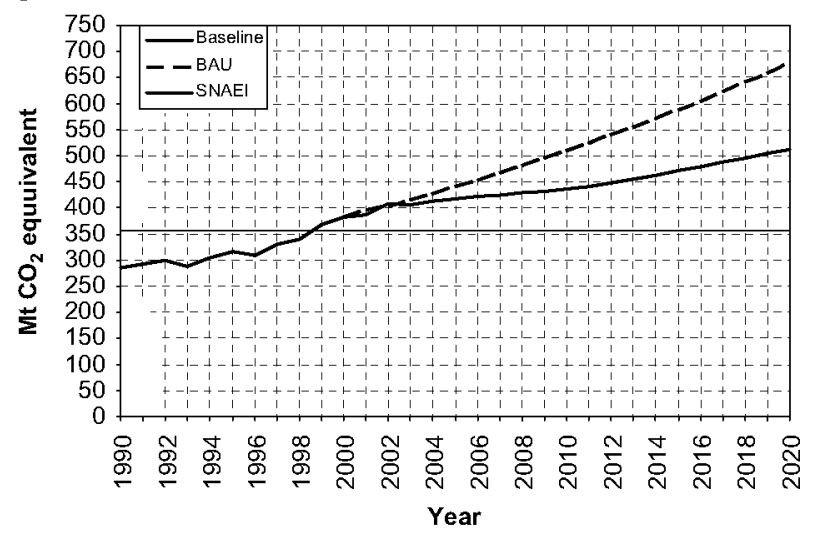

C

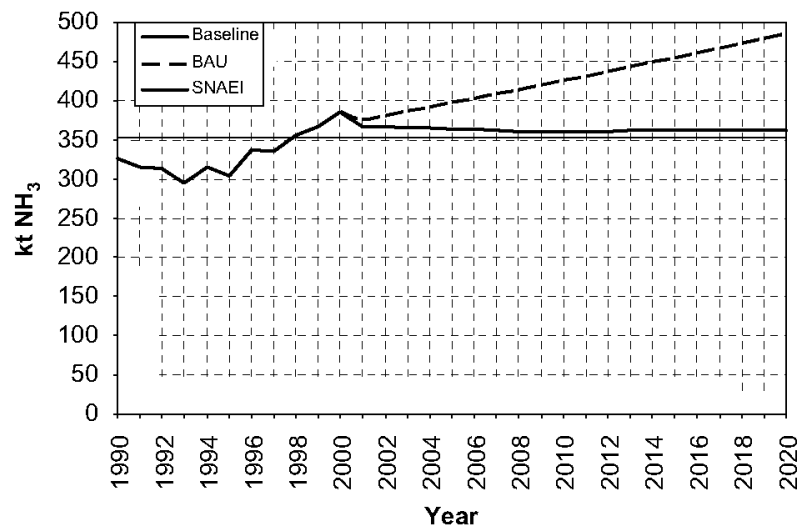

e

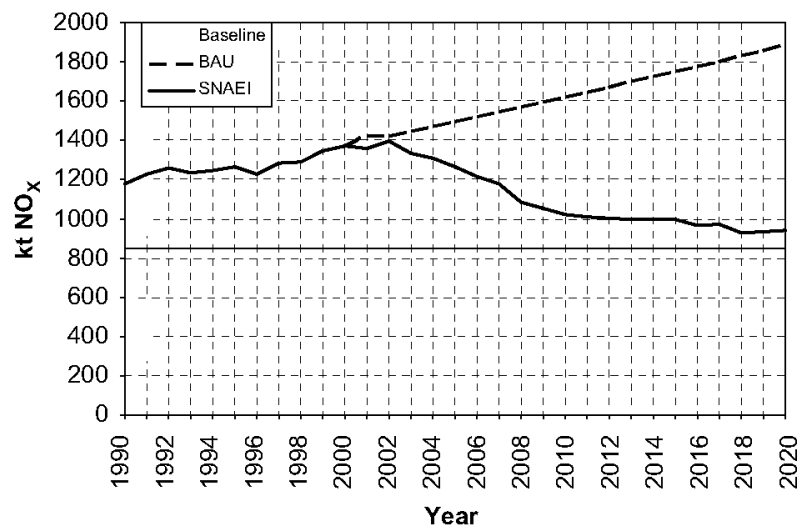

b

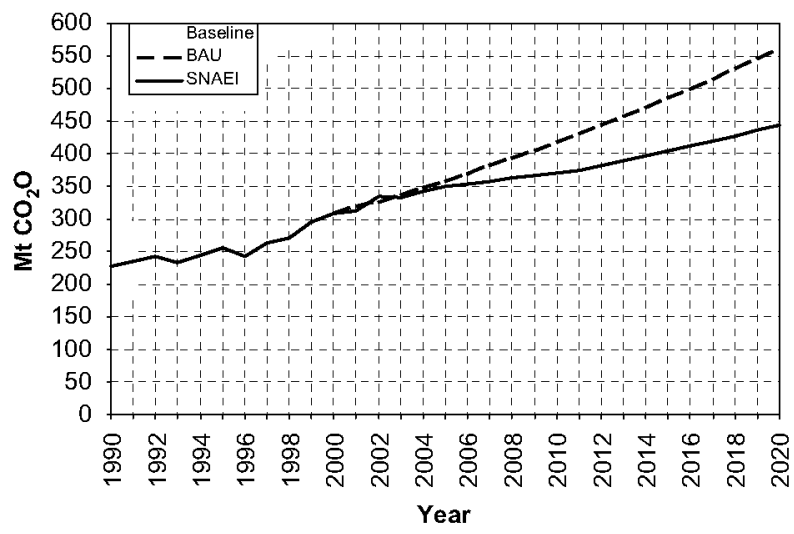

d

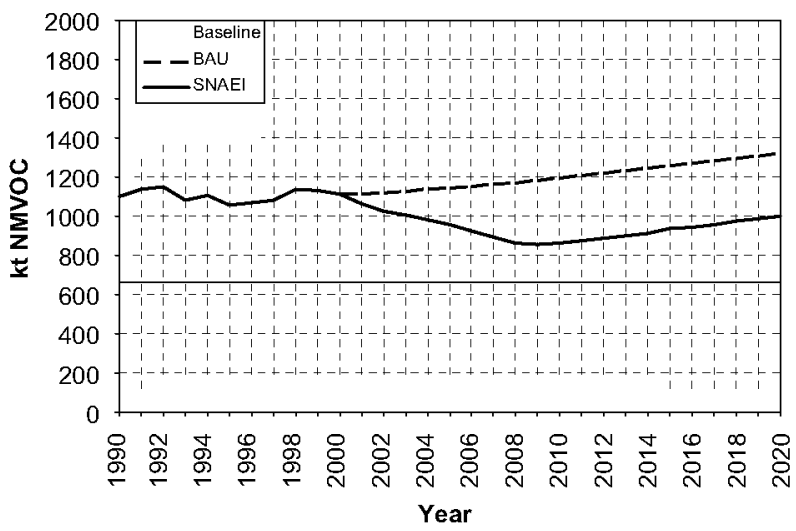

f

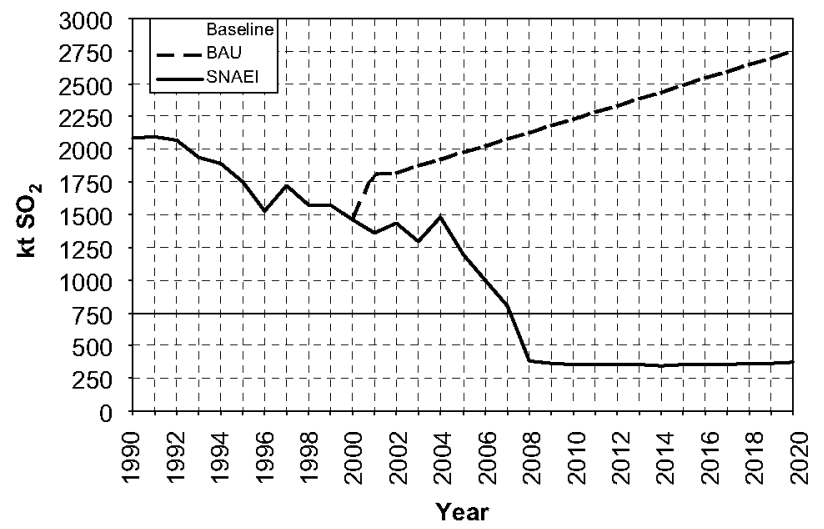

Fig. 5. Results for $\mathrm{CO}_{2}$, GHG (as $\mathrm{CO}_{2}$ equivalent), $\mathrm{NH}_{3}, \mathrm{NMVOC}, \mathrm{NO}_{x}$ and $\mathrm{SO}_{2}$. The black line represents official estimates for past years in accordance to the SNAEI.

$78 \mathrm{kt}(26 \%)$ while new directives and penetration of technologies on solvent use are capable to decrease emissions by $181 \mathrm{kt}$. For $\mathrm{NO}_{x}$ reduction, on-road transport measures have the highest reduction potential. The implementation of new engines will lead to a $232 \mathrm{kt}$ decrease in Spanish emissions (37\% of total reduction) while fuel switch and use of selective reduction systems on Power Plants reduce $222 \mathrm{kt}$. Measures for stationary sources such as desulphurization systems on power plants along 
Table 1

Effect of policies and measures included in the baseline scenario for 2010 compared to emissions under the BAU scenario

\begin{tabular}{|c|c|c|c|c|c|c|}
\hline \multirow{2}{*}{$\begin{array}{l}\text { SNAP } \\
\text { group }\end{array}$} & \multirow{2}{*}{$\begin{array}{l}\text { Policies and measures (numbers } \\
\text { according to Table } 1 \mathrm{~s} \text { in } \\
\text { supplementary material) }\end{array}$} & \multicolumn{5}{|c|}{ Atmospheric emission reductions (in kt except for GHG in $\mathrm{Mt}$ ) } \\
\hline & & GHG & $\mathrm{NH}_{3}$ & NMVOC & $\mathrm{NO}_{x}$ & $\mathrm{SO}_{2}$ \\
\hline 1 & $1-7$ and 9 & 27.7 & -0.1 & 0.2 & 222.3 & 1604.0 \\
\hline 2 & $1-4$ and $8-10$ & 2.5 & 0.0 & 5.7 & 10.2 & 3.6 \\
\hline 3 & $1-4$ and $9-16$ & 6.9 & 0.0 & 7.6 & 112.6 & 188.7 \\
\hline 4 & $3,7,10-11,13-14$, and $17-25$ & 3.7 & 7.1 & 66.4 & 6.8 & 33.2 \\
\hline 5 & $1-3$ and 23 & -0.7 & 0.0 & 0.7 & 0.0 & 0.0 \\
\hline 6 & $1-2,8,10,22,6-7$, and $26-28$ & 7.8 & 0.0 & 180.8 & 0.0 & 0.0 \\
\hline 7 & $1-4,9-10,22$, and $29-30$ & 8.5 & 4.2 & 78.4 & 232.0 & 24.3 \\
\hline 8 & $1-4,9,22,24$, and 31 & 2.2 & 0.0 & 4.5 & 49.6 & 24.3 \\
\hline 9 & $1-4,10,32-33$, and 35 & 6.1 & 1.8 & -4.1 & -1.9 & -1.0 \\
\hline 10 & $1-2,10,32$, and $34-36$ & 7.9 & 52.7 & 6.5 & -0.1 & 0.0 \\
\hline 11 & 10 and $34-36$ & 0.6 & -0.2 & -48.9 & -0.2 & 0.0 \\
\hline Total & $1-36$ & 73.0 & 65.6 & 297.7 & 631.5 & 1877.1 \\
\hline
\end{tabular}

Negative values mean that the combination of measures lead to an increase in emissions.



Fig. 6. Effect of P\&M included in the baseline scenario on Spanish $\mathrm{NO}_{x}$ emission projections within the EMEP domain.

with expected change in fuel mix would be remarkably effective in $\mathrm{SO}_{2}$ reduction, up to $1604 \mathrm{kt}(85 \%$ of national diminution).

Fig. 6 shows $\mathrm{NO}_{x}$ emission pattern for Spain as an example of annual reductions for the total temporal scope of the projections. In 2020, total savings would equal emissions, which reveals the magnitude of potential abatement of $\mathrm{P} \& \mathrm{M}$ on $\mathrm{NO}_{x}$ emissions.

The P\&M included not only consider technological options, but also determine the assumptions on key drivers such as energy consumption, industrial production, transport mobility, area occupied by different agricultural land uses, livestock, waste management, etc. A rather large increase in most socio-economic drivers for the period 2000-2020 is assumed: population growth $(21.2 \%)$, GDP $(77.6 \%)$ and annual household construction $(31.43 \%)$.

Fig. 5 shows the total national atmospheric emission projections for the main pollutants according to the inputs and assumptions under the baseline scenario. They are presented along with the BAU scenario and, when appropriate, with the Kyoto Protocol threshold or the National Emission Ceiling.

For GHG emission projections, the $\mathrm{CO}_{2}$ equivalent is expected to grow almost linearly with a high 
slope up to 2020 . The main reason for such a high increase is the expected GDP growth and energy consumption (MF, 2005; ME, 2006). The measures considered regarding energy saving and technology improvement are not able to counteract the increase of GHG main drivers. As a result, it is projected a $79.4 \%$ increase in emissions in 2020 (compared to 1990 ) while the BAU scenario would present a $137.4 \%$ increase. Hence, $\mathrm{P} \& \mathrm{M}$ included in baseline scenario allow a reduction of $165.9 \mathrm{Mt}$ of $\mathrm{CO}_{2}$ equivalent emissions in 2020. Considering GHG individually, only PFC emissions would be lower in 2020 than in $1990(-56.9 \%)$ due to a larger decrease in the use of PFC-14 than the increase in the use of PFC-218 and PFC-410. Changes in future emissions for the rest of the Kyoto Protocol gases are as follows: $+178.3 \%$ for $\mathrm{SF}_{6},+1287.5 \%$ for $\mathrm{HFC}$, $+94.0 \%$ for $\mathrm{CO}_{2},+17.5 \%$ for $\mathrm{CH}_{4}$ and $+14.1 \%$ for $\mathrm{N}_{2} \mathrm{O}$. The sharp increase expected in fluorinated gases pales, however, when compared to $\mathrm{CO}_{2}$ in terms of $\mathrm{CO}_{2}$ equivalent. Expected $\mathrm{CO}_{2}$ increment is mainly due to the growth of electricity demand, passenger mobility and freight transport. In relation to $\mathrm{CH}_{4}$, improvements on gas capture in landfills could not counteract the increase of waste generation per capita and population growth.

As for the NEC pollutants, only $\mathrm{NH}_{3}$ emissions will be higher in 2020 than in $1990(+10.9 \%)$ according to the baseline scenario. For NMVOC, $\mathrm{NO}_{x}$ and $\mathrm{SO}_{2} 2020$ emissions will be $8.8 \%, 20.5 \%$ and $82.4 \%$ lower than 1990 figures, respectively. These diminutions are due to the measures included in the baseline scenario likewise GHG (Table 1s and Table 1). Many of them imply the use of less polluting technologies (primary measures) or the penetration of abatement technologies (end-of-pipe measures). For instance, the national strategy for increasing renewable energies plans a $202 \%$ growth in wind power production, a $940 \%$ expansion of solar photovoltaic energy and a $15 \%$ rise in hydroelectricity production from 2004 to 2010 . Concerning abatement technologies, the national program for emission reductions on the power sector linked to the Large Combustion Plants Directive (2001/80/EC) foresee a broad implementation of flue gas desulphurization and selective catalytic reduction technologies to reduce $\mathrm{SO}_{2}$ and $\mathrm{NO}_{x}$ emissions, respectively. Nevertheless, they also include non-technical measures designed to modify people behaviour and to improve energy efficiency (e.g., the strategic plan for transport infrastructure (MF, 2005) introduces measures seeking to stimu- late a significant modal shift from road-based transport to rail-based transport such that the modal share of rail passengers is doubled by 2020). As for $\mathrm{NH}_{3}$, the increment is caused by expected growth of livestock (especially pigs and cattle), constant annually use of mineral fertilizer and changes in feed use and manure systems.

For the remaining pollutants (results not shown), the main results are as follows: PM is expected to rise in 2020 by $49.4 \%$ for TSP with respect to 1990 as a consequence of activity patterns expected for power plants, transport and agriculture. A higher increase trend is expected for $\mathrm{PM}_{10}$ and $\mathrm{PM}_{2.5}$ (174.9\% and $192.7 \%$, respectively). Regarding heavy metals, projections differ significantly: $\mathrm{Pb}$ emissions would be reduced by $92.2 \%$ mainly as a result of the mandatory decrease in gasoline lead contain; Cd will, approximately, maintain the 1990 levels (a decrease of $3.1 \%$ is expected) and $\mathrm{Hg}$ would be reduced by $9.1 \%$.

\section{Discussion}

\subsection{Consistency of emission projections}

The projection scheme presented in Section 2 allows the formulation of very specific, highly detailed hypotheses for each activity. This is an important advantage when performing the assessment of the measures assumed under any scenario, both from the technical and the cost-effectiveness points of view.

However, the main challenge confronted by this methodology is to ensure that individual emission projections have been aggregated in a consistent way into the superior hierarchical level (e.g., SNAP group, national total, etc.). As every SNAP activity is projected taking into account specific methods and assumptions, it is necessary to assure the compatibility and consistency of the different results. This is not a trivial question considering that activity rates are exogenous variables to the model that come from a wide range of information sources (macroeconomic models, population predictions, statistical methods, etc.) and that the demand forecast models that produce these estimates do not always consider the same basic socioeconomic inputs or driving forces such as future GDP, interest rates or population. The "Macroscenario" concept presented in Section 2.5 allows to overcome these obstacles since consistency across external variables can be checked. 


\subsection{Sensitivity analysis}

The European EMEP/CORINAIR programme has undertaken several initiatives to estimate the uncertainty from emission inventories distinguishing between validation and verification procedures in order to increase the quality of those emission compilations (EEA, 2006). Regarding GHG emissions, the uncertainty has been quantified by deriving probabilistic confidence intervals for the central emission estimates (UNFCC, 2000). The quantitative uncertainty analysis has been based either on Monte Carlo analysis or on a deterministic calculation of extreme emission values related to plausible ranges of input variables (Vardoulakis et al., 2002).

There are two basic methods to face the problem of uncertainty in forecasting: scenario analysis (developing scenarios that span an interesting range of possible outcomes) and uncertainty analysis (associating probabilities with outcomes) (Webster et al., 2002). For the CEP model, the first option has been adopted. However, a tool is currently being developed to quantify the total uncertainty in emission projections as a combination of different specific uncertainties related to the steps involved in the emission estimations. Information about the methodology will be published shortly.

In this study, the method selected identifies the critical CEP model parameters and the emission sources that contribute the largest amount of uncertainty in projected values. Investing time in determining likely ranges for unimportant parameters must be avoided (van der Sluijs et al., 2003). For this study, a three-step methodology has been developed. The steps are as follows: (i) identification of activities with higher emissions, (ii) in-depth examination of critical parameters for each such activity, and (iii) application of a unique percentage change to each critical parameter to obtain the results in order to find out their influence in emission projections.

For the application to Spain, the 20 most important activities were identified in the first step, which are those with a contribution $>5 \%$ of the total national emissions for any of the pollutants included in the SNAEI (Table 2). Following this approach, at least $70 \%$ of the total emissions for each pollutant are covered in the analysis except for NMVOC and $\mathrm{N}_{2} \mathrm{O}$, which are mostly emitted by diffuse sources. Second, 41 parameters were found to be the critical uncertainty drivers for each activity

Table 2

Top 20 activities for sensitivity analysis including their percentage of total 2000 emissions

\begin{tabular}{|c|c|c|c|c|c|c|c|c|c|c|c|}
\hline No. & SNAP code & $\mathrm{SO}_{x}$ & $\mathrm{NO}_{x}$ & NMVOC & $\mathrm{CH}_{4}$ & $\mathrm{CO}_{2}$ & $\mathrm{~N}_{2} \mathrm{O}$ & $\mathrm{NH}_{3}$ & $\mathrm{SF}_{6}$ & $\mathrm{HFC}$ & $\mathrm{PFC}$ \\
\hline 1 & 01.01 .01 & 63.6 & 17.9 & 0.2 & - & 27.7 & 0.9 & - & - & - & - \\
\hline 2 & 02.02 .02 & 1.1 & 1.2 & 1.5 & 1.6 & 5.4 & 0.7 & - & - & - & - \\
\hline 3 & 03.01 .03 & 4.9 & 1.8 & 0.1 & 0.1 & 5.8 & 0.5 & - & - & - & - \\
\hline 4 & 03.03 .11 & 3.5 & 3.7 & - & - & 3.2 & 0.3 & - & - & - & - \\
\hline 5 & 04.03 .01 & 0.3 & 0.1 & - & - & 0.2 & - & - & - & - & 90.3 \\
\hline 6 & 04.06 .11 & - & - & 4.5 & - & - & - & - & - & - & - \\
\hline 7 & 04.06 .12 & - & - & - & - & 4.9 & - & - & - & - & - \\
\hline 8 & 04.08 .01 & - & - & - & - & - & - & - & - & 36.0 & - \\
\hline 9 & 06.05 .02 & - & - & - & - & - & - & - & - & 44.3 & 9.1 \\
\hline 10 & 06.05 .06 & - & - & - & - & - & - & - & - & 15.3 & - \\
\hline 11 & 06.05 .07 & - & - & - & - & - & - & - & 100.0 & - & - \\
\hline 12 & $07^{\mathrm{a}}$ & 1.1 & 37.3 & 7.4 & 0.5 & 25.0 & 6.5 & 1.4 & - & - & - \\
\hline 13 & 07.06 .00 & - & - & 3.4 & - & - & - & - & - & - & - \\
\hline 14 & 08.06 .00 & 0.1 & 7.2 & 0.6 & - & 2.2 & 0.2 & - & - & - & - \\
\hline 15 & 09.04 .01 & - & - & - & 15.4 & - & - & - & - & - & - \\
\hline 16 & 10.01 .02 & - & 0.6 & 6.1 & - & - & 23.5 & 38.1 & - & - & - \\
\hline 17 & 10.01 .05 & - & 0.1 & - & - & - & 16.8 & 12.1 & - & - & - \\
\hline $18^{\mathrm{b}}$ & $10.0 \mathrm{X}$ & - & - & - & 25.3 & - & - & 5.6 & - & - & - \\
\hline $19^{\mathrm{b}}$ & $10.0 \mathrm{X}$ & - & - & - & 21.2 & - & - & 14.9 & - & - & - \\
\hline $20^{\mathrm{b}}$ & $10.0 \mathrm{X}$ & - & - & - & 12.3 & - & - & 0.7 & - & - & - \\
\hline \multicolumn{2}{|c|}{ Total $(\%)$} & 74.5 & 69.9 & 23.8 & 76.4 & 74.5 & 49.3 & 72.9 & 100.0 & 95.6 & 99.5 \\
\hline
\end{tabular}

${ }^{a}$ Except 07.06 (gasoline evaporation) and 07.07 (tyre and brake wear).

${ }^{\mathrm{b}} 18,19$ and 20 correspond to dairy cows, fattening pigs and ovines, respectively. 
(Table 3 ). Finally, a $\pm 10 \%$ variation was applied to these parameters.

\subsection{Comparison with RAINS/GAINS}

Due to the reporting requirements imposed by the NEC Directive and the Kyoto Protocol, most European countries have produced national emission projections which are currently being used as inputs to the continental scale Regional Air Pollution INformation and Simulation (RAINS) model. This system is intended to identify cost-optimal allocations of emission reductions in Europe (Amann et al., 2004) compatible with the deposition and concentration targets for the protection of human health and ecosystems determined in the Gothenburg Protocol and the European Thematic Strategy on Air Pollution.
The RAINS model consists of several modules that provide national emission projections and costs related to different end-of-pipe control measures in future years. The results are eventually used to determine regional air quality levels at $50 \mathrm{~km} \times 50 \mathrm{~km}$ resolution (EMEP, 2004). In recent years, the RAINS model has been updated into the Greenhouse Gas-Air Pollution Interactions and Synergies (GAINS) model (Klaassen et al., 2004), which also considers structural and non-technical measures.

The main differences between the methodologies implemented in GAINS and CEP to estimate future emissions are the level of detail in activity disaggregation and the associated input data requirements. GAINS uses lower resolution data that ensure the consistency of Europe-wide emission projections. These input data are obtained from a

Table 3

Main uncertainty-related parameters for each activity (top 20)

\begin{tabular}{|c|c|c|c|}
\hline No. & SNAP code & Activity name & Parameters \\
\hline 1 & 01.01 .01 & Public power combustion plants $\geqslant 300 \mathrm{MW}$ & Fuel consumption, gas consumption \\
\hline 2 & 02.02 .02 & Residential combustion plants $<50 \mathrm{MW}$ & Fuel consumption, gas consumption \\
\hline 3 & 03.01 .03 & $\begin{array}{l}\text { Combustion in manufacturing industry plants } \\
<50 \mathrm{MW}\end{array}$ & Fuel consumption, gas consumption \\
\hline 4 & 03.03 .11 & Combustion in cement production & Fuel consumption, $\mathrm{SO}_{2}$ and $\mathrm{NO}_{x} \mathrm{EF}$ \\
\hline 5 & 04.03 .01 & Aluminium production (electrolysis) & Al production, production technology \\
\hline 6 & 04.06 .11 & Road paving with asphalt & $\begin{array}{l}\text { Asphalt production, asphalt tones per } \mathrm{km} \\
\text { paving, } \mathrm{km} \text { paved }\end{array}$ \\
\hline 7 & 04.06 .12 & Cement production (decarbonizing) & Cement production \\
\hline 8 & 04.08 .01 & $\begin{array}{l}\text { Halogenated hydrocarbons production. By- } \\
\text { products }\end{array}$ & $\begin{array}{l}\text { Production of halogenated hydrocarbons, } \\
\text { plants location }\end{array}$ \\
\hline 9 & 06.05 .02 & $\begin{array}{l}\text { Refrigeration and air conditioning equipments } \\
\text { using halocarbons }\end{array}$ & $\begin{array}{l}\text { Sales of equipments, lost in charge }(k) \text {, lost } \\
\text { during lifetime }(x)\end{array}$ \\
\hline 10 & 06.05 .06 & Aerosol cans & $\begin{array}{l}\text { Consumption of cans, rate of gas injected per } \\
\text { can }\end{array}$ \\
\hline 11 & 06.05 .07 & $\begin{array}{l}\text { Electrical equipments (except electronic } \\
\text { components manufacturing) }\end{array}$ & $\begin{array}{l}\text { Stock amount, EF during equipment's } \\
\text { manufacturing, installation, operating phase, } \\
\text { and disposal }\end{array}$ \\
\hline 12 & 07 & $\begin{array}{l}\text { Road transport (except Gasoline evaporation } \\
\text { and tyre and brake wear) }\end{array}$ & $\begin{array}{l}\text { Mileage travelled by cars, light- and heavy-duty } \\
\text { vehicles and motorcycles }\end{array}$ \\
\hline 13 & 07.06 .00 & Gasoline evaporation from vehicles & $\begin{array}{l}\text { Mileage travelled by cars, light- and heavy-duty } \\
\text { vehicles and motorcycles }\end{array}$ \\
\hline 14 & 08.06 .00 & $\begin{array}{l}\text { Other mobile sources and machinery in } \\
\text { agriculture }\end{array}$ & $\begin{array}{l}\text { Number of agricultural machinery, number of } \\
\text { usage hours per year, Horsepower average }\end{array}$ \\
\hline 15 & 09.04 .01 & Managed waste disposal on land & $\begin{array}{l}\text { Amount of MSW to landfills, organic content, } \\
\text { paper and cardboard content }\end{array}$ \\
\hline 16 & 10.01 .02 & Permanent crops with fertilizers & $\begin{array}{l}\text { Dose of organic and inorganic fertilizer applied } \\
\text { to the crops }\end{array}$ \\
\hline 17 & 10.01 .05 & Grassland with fertilizers & $\begin{array}{l}\text { Dose of organic, inorganic and total fertilizer } \\
\text { applied to the crops }\end{array}$ \\
\hline 18 & $10.0 \mathrm{X}$ & Dairy cows & Number of cows, emission factors \\
\hline 19 & $10.0 \mathrm{X}$ & Fattening pigs & Number of pigs, emission factors \\
\hline 20 & $10.0 \mathrm{X}$ & Ovines & Number of ovines, emission factors \\
\hline
\end{tabular}


variety of European models: economic models such as PRIMES for Energy system model for EU27+3 countries (Capros and Mantzos, 2004) and other sector-specific models such as TREMOVE/ SCENES which is used for the impact assessment of transport policies for EU21 (De Ceuster et al., 2006) and CAPRI that models and assess EU27 agricultural policies (Heckelei and Britz, 2000). In addition to this, GAINS has developed national scenarios for each EU Member State based on their own activity rate projections.

On the other hand, CEP uses more detailed emissions disaggregation and hypotheses for future developments, as well as a higher spatial resolution of input data. CEP is capable of accurately simulating future emission scenarios for each activity sector in a given country, due to the specificity of input data. CEP includes an original nomenclature to compare emission and activity rates estimations with GAINS results and vice versa based on a fourth hierarchical level for the original SNAP nomenclature.

The comparison between baseline scenarios (Base and NEC_NAT_CLE) shows reasonable agreement. Trends are rather consistent for all the pollutants, especially $\mathrm{NH}_{3}$ and $\mathrm{CO}_{2}$. Maximum differences were observed to be $10 \%$ for $\mathrm{NO}_{x}$ and $30 \%$ for $\mathrm{SO}_{x}$ in the year 2010 . These values were reduced to $9 \%$ and $18 \%$, respectively, in the year 2020 .

\section{Conclusions}

In this paper, a methodology (CEP) to evaluate national emission projections in a consistent way has been presented. Following this methodology, emission projections are calculated for different scenarios depending on national socio-economic, legislative and technological aspects. This methodology was applied to Spain to evaluate all pollutants covered by the Geneva Convention and the Kyoto Protocol, from all emission sources included in the SNAP nomenclature. The model, however, might be applied to project any other pollutant.

The innovative aspects of CEP include a fully consistent approach for estimating emission projections across a wide range of national activity sectors. The high level of detail used in the calculations allows a further temporal and spatial disaggregation of emission projections for air quality modelling purposes including the assessment of fulfilment of standards and comparison with EMEP/RAINS results.

A three-step sensitivity analysis methodology has been developed as a first step to projections uncertainty assessment. The 20 most polluting activities were identified along with their main driver parameters. A variation of the parameters led to different scenario that permits an analysis of their influence in the emissions. However, future efforts should be oriented to perform an advanced uncertainty analysis.

In addition, a tailored software tool (EmiPro) has been produced to support data management, quality checks, quality assurance, and report generation. The flexibility of the methodology and associated tool enable decision-makers to assess different emission control policies (e.g., abatement technologies) in a straightforward and comprehensive manner.

A specific nomenclature (CEP) has been designed to compare emission projections classified on SNAP, CRF or NFR to RAINS/GAINS nomenclature. It contents 1439 activities and a mapping with their biunivocal relation with RAINS activities. This mapping allows a comparison of activity rate and emission estimates from both models and may be adopted by any country.

CEP has been applied to Spain for the period 2001-2020. The analysis of results showed a substantial increase in atmospheric emissions for the BAU scenario while P\&M included in the baseline scenario led to lower increments up to 2020. The differences between both scenarios corresponded to the effect of P\&M likely to be implemented in the future. It was found that measures on electricity generation (energy savings, desulphurization technologies, Selective Catalytic Reductions, changes in the fuel mix, etc.) are very effective for $\mathrm{GHG}, \mathrm{NO}_{x}$ and $\mathrm{SO}_{2}$ (responsible of $38 \%, 35 \%$ and $85 \%$ of total reductions, respectively) while P\&M in solvent use and road transport are crucial to reduce NMVOC $(61 \%$ and $26 \%)$. Agriculture initiatives determine $80 \%$ of $\mathrm{NH}_{3}$ improvements and transport measures (shift from road transport to rail, further implementation of reduction technologies in road transport vehicles, etc.) contribute to $45 \%$ of total $\mathrm{NO}_{x}$ abatements.

Finally, this study demonstrates that the application of a very detailed methodology for atmospheric emission projections in a country provide improved results than less in-depth evaluations and can help identifying most efficient measures to reduce 
emissions, assessing legislation compliances, and supporting decision-making. Future work should focus on extended uncertainty analysis, calculation of cost/effectiveness ratios for each measure, adaptation of outputs to be used as input for air quality modelling, and linking projections with health and environmental impacts (e.g., by using projections for modelling air quality and its effects).

The methodology presented may be adapted to any particular region for different purposes. Nonetheless, it was designed in consistency with the EMEP/CORINAIR methodology and the CAFE framework and therefore it may be easily exported to any European country to be applied on a routinary basis.

\section{Appendix A. Supplementary materials}

Supplementary data associated with this article can be found in the online version at doi:10.1016/ j.atmosenv.2008.02.067.

\section{References}

Amann, M., Cofala, J., Heyes, C., Klimont, Z., Mechler, R., Posch, M., Schöpp, W., 2004. The RAINS model. Documentation of the model approach prepared for the RAINS peer review 2004. Interim Report. International Institute for Applied Systems Analysis.

Amann, M., Asman, W., Bertok, I., Cofala, J., Heyes, C., Klimont, Z., Schöpp, W., Wagner, F., 2007. Updated Baseline Projections for the Revision of the Emission Ceilings Directive of the European Union. Report from the International Institute for Applied Systems Analysis (IIASA).

Borge, R., Lumbreras, J., Rodriguez, E., Casillas, I., 2005. Supporting Spain's national emission projections with the EmiPro tool. In: Proceedings of the 14th International Emission Inventory Conference: "Transforming Emission Inventories-Meeting Future Challenges Today," Las Vegas, USA.

Capros, P., Mantzos, L., 2004. "The PRIMES Energy System Model," model manual. Available from: 〈www.e3mlab.ntua.gr/manuals/PRIMsd.pdf $>$.
CITEPA, 2003. Expert Group on Techno-Economic Issues (EGTEI). Methodological aspects and sectoral documents. 〈http://www.citepa.org/forums/egtei/egtei_index.htm〉.

De Ceuster, G., van Herbruggen, B., Logghe, S., Ivanova, O., Carlier, K., 2006. TREMOVE 2, Final Report. Part 1: Description of Model Version 2.44. European Commission, DG ENV. Available from: 〈http://www.tremove.org/documentation/TREMOVE_2.44_model.pdf $\rangle$.

Derwent, R.G., Simmonds, P.G., O'Doherty, S., Stevenson, D.S., Collins, W.J., Sanderson, M.G., Johnson, C.E., Dentener, F., Cofala, J., Mechler, R., Amann, M., 2006. External influences on Europe's air quality: baseline methane, carbon monoxide and ozone from 1990 to 2030 at Mace Head, Ireland. Atmospheric Environment 40, 844-855.

EC, 2004. European Pollutant Emission Register Report.

EEA, 2000. COPERT III-computer programme to calculate emissions from road transport. Methodology and emission factors (Version 2.1). Available from: 〈http://reports.eea.europe.eu/ Technical_report_No_49 $>$ (EEA Technical Report No. 49).

EEA, 2006. EMEP/CORINAIR Emission Inventory Guidebook-2006. Available from: <http://reports.eea.europe.eu/ EMEPCORINAIR4) (EEA Technical Report No. 30 ).

EMEP, 2004. Status Report. Transboundary Acidification, Eutrophication and Ground Level Ozone in Europe. Joint MSC-W \& CCC \& CIAM \& ICP-M\&M \& CCE Report.

Garg, A., Shukla, P.R., Kapshe, M., Menon, D., 2004. Indian methane and nitrous oxide emissions and mitigation flexibility. Atmospheric Environment 38, 1965-1977.

GC, 1979. Convention on long-range transboundary air pollution. Signed in November 1979.

Heckelei, T., Britz, W., 2000. Concept and explorative application of an EU-wide. Regional Agricultural Sector Model (CAPRI-Project). In: Paper presented at "65th EAAE Seminar, Agricultural Sector Modelling and Policy Information Systems, Bonn, Germany, 29-31 March, 2000.

IVL, 2006. Workshop on the importance of non-technical measures for reductions in emission of air pollutants and how to consider them in integrated assessment modelling. Available from: 〈www.ivl.se〉.

Klaassen, G., Amann, M., Berglund, C., Cofala, J., HöglundIsaksson, L., Heyes, C., Mechler, R., Tohka, A., Schöpp, W., Winiwarter, W., 2004. The Extension of the RAINS Model to Greenhouse Gases. IR-04-015. International Institute for Applied Systems Analysis (IIASA), Laxenburg, Austria.

Klimont, Z., Streets, D.G., Gupta, S., Cofala, J., Lixin, F., Ichikawa, Y., 2002. Anthropogenic emissions of non-methane volatile organic compounds in China. Atmospheric Environment 36, 1309-1322.

McCulloch, A., Lindley, A.A., 2007. Global emissions of HFC23 estimated to year 2015. Atmospheric Environment 41, $1560-1566$.

ME (Ministerio de Economía), 2006. Informe de planificación de los sectores de electricidad y gas 2002-2011. 2006 update for the period 2005-2011.

MF (Ministerio de Fomento), 2005. Plan Estratégico de Infraestructuras y Transporte (PEIT) 2005-2020.

MMA, 2006. Spain's National Atmospheric Emission Inventory. Ministry of Environment (MMA).

Pirrone, N., Costaa, P., Pacynab, J.M., Ferrara, R., 2001. Mercury emissions to the atmosphere from natural and anthropogenic sources in the Mediterranean region. Atmospheric Environment 35, 2997-3006. 
Samaras, Z., Zachariadis, T., Holtmann, T., Rentz, O., Zierock, K.-H., 1999. A methodology and a database for forecasting anthropogenic atmospheric emissions in Europe. Atmospheric Environment 33, 3389-3404.

Streets, D.G., Waldhow, S.T., 2000. Present and future emissions of air pollutants in China: $\mathrm{SO}_{2}, \mathrm{NO}_{x}$ and $\mathrm{CO}$. Atmospheric Environment 34, 363-374.

UNFCC, 2000. Good Practice Guidance and Uncertainty Management in National Greenhouse Gas Inventories.

UNFCCC, 1997. Kyoto Protocol to the United Nations Framework Convention on Climate Change. United Nations. Available from: 〈http://unfccc.int/resource/docs/convkp/kpeng.pdf $\rangle$.

US EPA, 1999. Emission Projections (Volume X). Prepared by "The Pechan-Avanti Group" for the US EPA.

USA, 2002. US Department of State, US Climate Action Report 2002, Washington, DC. van der Sluijs, J.P., Risbey, J.S., Kloprogge, P., Ravetz, J.R., Funtowicz, S.O., Quintana, S.C., Pereira, S.G., de Marchi, B., Petersen, A.C., Janssen, P.H.M., Hoppe, R., Huijs, S.W.F., 2003. RIVM/MNP Guidance for Uncertainty Assessment and Communication. Utrecht University.

van Harmelen, A.K., Pulles, M.P.J., 2000. From Inventory to Policy: Outline for a General Methodology for the Development of Emission Projections. TNO Institute.

Vardoulakis, S., Fisher, B.E.A., Gonzalez-Flesca, N., Pericleous, K., 2002. Model sensitivity and uncertainty analysis using roadside air quality measurements. Atmospheric Environment 36, 2121-2134.

Webster, M.D., Babiker, M., Mayer, M., Reilly, J.M., Harnisch, J., Hyman, R., Sarofim, M.C., Wang, C., 2002. Uncertainty in emissions projections for climate models. Atmospheric Environment 36, 3659-3670. 